\title{
Special issue on recent advancements in machine learning algorithms for internet of things
}

\author{
Gunasekaran Manogaran ${ }^{1} \cdot$ Naveen Chilamkurti $^{2}$. \\ Ching-Hsien $\mathrm{Hsu}^{3}$
}

Published online: 5 June 2018

(C) Springer Science+Business Media, LLC, part of Springer Nature 2018

In recent years, wireless sensor networks are continuously generating a massive amount of data. Processing this huge amount of data is not possible by traditional tools and technologies. Hence, there is a need for scalable machine learning algorithms to process such massive amount of data. In recent years, scalable machine learning algorithms are developed to process the massive IoT data. This special issue focused on innovations in scalable machine learning algorithms and embedded system development.

The first paper, entitled QOS Distributed Routing Protocol for Mobile Ad-Hoc Wireless Networks Using Intelligent Packet Carrying Systems, by Murugeswari and Rathi, proposes an intelligent packet carrying algorithm; it provides a tracking mechanism that tracks nodes in rural places. The effectiveness and reliability has been calculated and the results are obtained using OPNET simulator.

The second paper, entitled TEE based Session Key Establishment Protocol for Secure Infotainment Systems, by Sungbum Lee and Jong-Hyouk Lee, proposes a session key establishment protocol using Elliptic Curve Cryptography. The proposed protocol enables secure authentication and key distribution between a user device and a telematics control unit.

Third paper, entitled A novel Gini Index Decision Tree Data Mining Method with Neural Network Classifiers for Prediction of Heart Disease, by K. Mathan et al., proposes an altered

\footnotetext{
$凶$ Gunasekaran Manogaran

gunavit@gmail.com

Naveen Chilamkurti

n.chilamkurti@latrobe.edu.au

Ching-Hsien Hsu

chh@chu.edu.tw

1 University of California, Davis, USA

2 LaTrobe University, Melbourne, Australia

3 Chung Hua University, Hsin-chu, Taiwan
} 
calculation for classification with decision trees. This work is planned to show the data mining method in disease forecast frameworks in medicinal space by selecting the best attributes. This paper demonstrates that among various prediction models neural networks and Gini index prediction models results with most noteworthy precision for heart attack prediction.

Fourth paper, entitled A New Deep Spatial Transformer Convolutional Neural Network for Image Saliency Detection, by Xinsheng et al., proposes a novel deep spatial transformer convolutional neural network (Spatial Net) framework for the detection of salient and abnormal areas in images. This paper introduces spatial attention into CNN layers to extract global and local features of each image sample. Global feature information is used in this paper to highlight the features of internal salient regions and local feature information mainly helps to control background noise.

Fifth paper, entitled A Hybrid Approach of Neutrosophic Sets and DEMATEL Method for Developing Supplier Selection Criteria, by Mohamed Abdel-Basset et al., presents a study on the Neutrosophic Set for Decision Making and Evaluation Method (DEMATEL) to analyze and determine the factors influencing the selection of Supply Chain Management (SCM) suppliers. This research was designed by Neutrosophic DEMATEL data collection survey of experts, interviewing professionals in management, procurement and production.

We would like to convey our sincere thanks to all the researchers for submitting their manuscripts and a special note of thanks to the reviewers, whose efforts have allowed the selection of good quality papers. We are also grateful to the Journal of Design Automation for Embedded Systems, for allowing us to divulge a selected sample of the ongoing research efforts on recent advancements in machine learning algorithms especially in IoT applications. 